\title{
Using AWS Cloud resources in Operating Systems undergraduate class during the pandemic: a case study
}

\author{
André Leon S. GradvohI 1,2,3 \\ ${ }^{1}$ University of Campinas, Limeira-SP, Brazil \\ ${ }^{2}$ AWS Educate Cloud Faculty Ambassador \\ ${ }^{3}$ IEEE Senior Member \\ gradvohl@ft.unicamp.br
}

June 25, 2020

1 andemic brought new forms of remote education into the discussion. However, facilitating students' access to good computing infrastructure is not a widespread task. This paper presents a report on the use of computing resources in the AWS cloud by students in the Operating Systems class during the pandemic, from March to July 2020. The use of these computational resources was essential to consolidate some of the concepts covered in the course and, at the same time, to complement the students' knowledge about cloud computing. The results of this survey were very positive. Students said they learned more about the resources available in the cloud, the potential of cloud computing, and how to use it. Besides, they were able to create their remote infrastructure to carry out the work proposed in the Operating System class.

\section{Introduction}

It is necessary to innovate and look for possibilities to continue offering quality education to students in the dark times we are living, dealing with social distance and with the impossibility of having faceto-face classes. In Brazil, in particular, undergraduate students do not always have access to reasonable computational resources to develop their work. In some cases, the Internet connection itself is weak, making it difficult even to take classes online.

In short, there are many difficulties. However, some companies have collaborated 
with universities in the search for solutions that alleviate the difficulties of connection and use of computational resources. One such initiative is the AWS Educate program.

In this paper, we report an experience used in the Operating Systems class, offered in the first semester of 2020 (March to July 2020), for the Information Systems undergraduate course at the School of Technology at the State University of Campinas (UNICAMP). The experience was very positive for the students, as they were able to have computational resources remotely for the class's work. Besides, they were also able to set up their environment to develop of the work of other classes.

The paper adopts the following structure. Section 2 defines the context of the Operating Systems class and the need to propose a work that uses the computational resources of the AWS cloud. Section 3 comments on the AWS Educate program and how it helped the class. Section 4 describes the work proposal for the Operating Systems class. Section 5 shows the results of an evaluation of students' use of AWS and Section 6 discusses these results. Finally, Section 7 presents the conclusions.

\section{Operating Systems class context}

In its regular offering, the Operating Systems class has about $2 / 3$ of theoretical activities - where the professor discusses and explores the concepts - and $1 / 3$ of practical activities. In the theoretical part, the professor explores process management, memory, virtual memory management, input and output, device management, file systems management, virtualization, and security.

In the practical part, he addresses the creation of processes and threads, synchronization mechanisms, classic synchronization problems, interruptions and signal processing, and basic administration of operating systems. In this practical part, the use of computer labs is essential for students to experience the application of concepts in real situations or the closest to that.

Before the pandemic, students could have access to the equipment of the university's laboratories, properly prepared for the proposed activities, without fear of damaging any facility. With the pandemic and often having equipment that is shared with the rest of the family or without the necessary installation to carry out the experiments, students were limited.

\section{The AWS Educate Program}

In this sense, the AWS Educate Program was essential. It is a program that provides students with resources for developing cloudrelated skills. The program facilitates access to AWS content, training services, and the AWS Educate Job Board with employment opportunities. Furthermore, professors can access AWS services, start virtual classrooms, and use tools to help students learn about the cloud.

In the particular case of the Operating Systems discipline, we mainly use AWS computational services. For the class, we requested a virtual classroom and credits in the amount of US\$ 50.00 for each student enrolled in the class to carry out their work in the cloud. It sums US $\$ 1,600.00$ specifically for that class. We covered the work that students would need to develop and their relationship with the class in Section 4.

The creation of the remote virtual room allowed us to follow the work that the students developed and their engagement in the discipline. At the same time, students had the necessary autonomy to carry out their experiments in a computational environment that they specified, without fear that these experiments would damage their local system. After all, in the worst case, the remote infrastructure could be redone in a short time, with a few steps. 


\section{The work proposal for the class}

To compose the grade for the class, beyond the regular tests, we proposed an academic work using AWS computational resources. In a nutshell, the work was to create an instance on the Amazon Elastic Compute Cloud (EC2), with a specific configuration. We proposed that students do that work in pairs.

Before students created their instance, they should have calculated the costs of the instance. Each student had only US\$ 50.00. Therefore, they should keep the costs low, so that there is time for the professor to evaluate the work.

After creating their instance, they should perform other work on the class in that instance. Among these works, there was the creation of processes and multiple threads to solve some computational problems, which included synchronization techniques.

In addition to creating and managing the instance, students needed to ensure that remote connections to the instance - in particular the professor's connections - are secured.

All steps for creating the instance, its administration, security issues, and even the execution of the solution of the proposed problems using the concepts of multithreads, synchronization, and interruptions, should be recorded on video. The video could be published on YouTube or another repository that could be accessed by the professor.

An important point that deserves to be highlighted is that the work should be carried out in the most autonomous way possible. In specifying the work, the professor asked the students to try to solve the work by seeking the documentation available on the AWS itself, using the professor only as a last resort, i. e. if the doubt was not resolved with the available documentation. In this sense, there were rare occasions when students had doubts. Even so, the doubts presented were more related to the implementation of the concepts of threads and synchronization than to the AWS infrastructure.

Some videos produced by the students were so good that they can serve as tutorials for other interested people. Even if the viewer is not an Operating Systems student, the videos provide step-by-step instructions for creating the user's own AWS instance from the AWS Educate Program.

\subsection{The video}

Several groups produced their videos with all the development of the work, from the creation of the instance to the configuration and administration of the system to the remote and secure connection by the professor. The video also showed the execution of the solution to the proposed problem using multiple threads.

The video illustrated in Figure 1, available at YouTube, is one of the best works produced in the discipline. Although the audio in the video is in Brazilian Portuguese, we invite the reader to watch it.

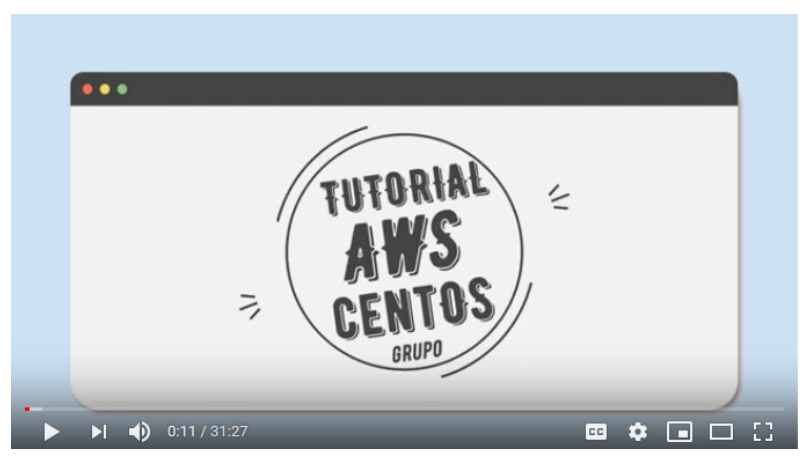

Figure 1: One of the best videos produced as a result of the work.

\section{Assessing the use of AWS Cloud resources}

To assess the impact of using AWS Cloud resources in the Operating Systems class, we carried out a survey with 20 students enrolled and who completed the class. In this survey, we asked simple questions about the relevance 
Using AWS Cloud resources in Operating Systems undergraduate class during the pandemic: a case study

of the work for the class, about the difficulties the students faced, and the knowledge they obtained when doing the work. We also asked about the continued use of the AWS Educate Program after the discipline.

We present the results in the following sections.

\subsection{Relevance of work for the class}

We questioned the students about how pertinent the work was to the discipline. The goal was to verify how well the students perceived the practical application of the concepts covered in the discipline. Figure 2 illustrates the result.

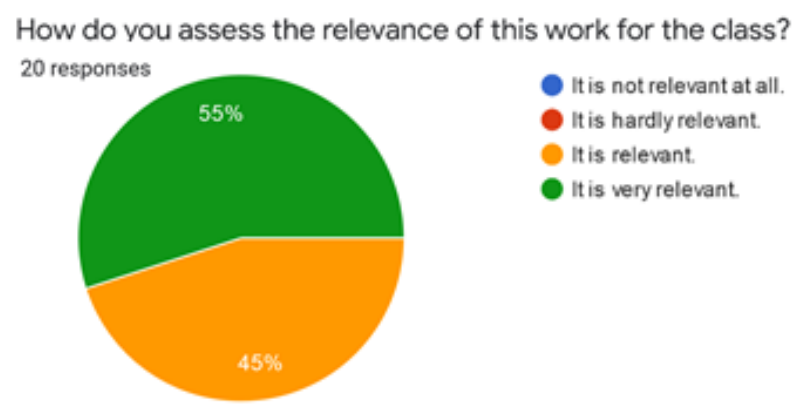

Figure 2: The relevance of work for the class.

Thus, $55 \%$ of the respondents considered it relevant, and the other $45 \%$ considered it is very relevant. Nobody claimed that it was irrelevant.

\subsection{Difficulties faced to carry out the work}

In this issue, the goal was to determine if the students faced many difficulties when doing the work and if these difficulties prevented them from doing the work. Figure 3 shows the result.

In this matter, all the students faced difficulties (many or few), but all managed to overcome them.

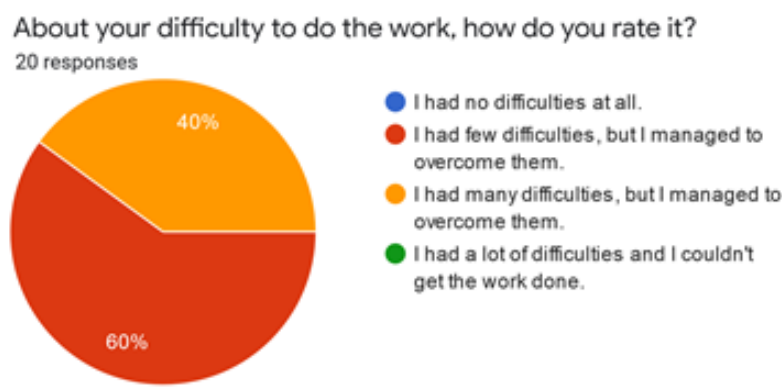

Figure 3: Difficulties faced to carry out the work.

\subsection{Knowledge obtained in carrying out the work}

We questioned the students if they gained new knowledge when doing the work. Figure 4 shows the result.

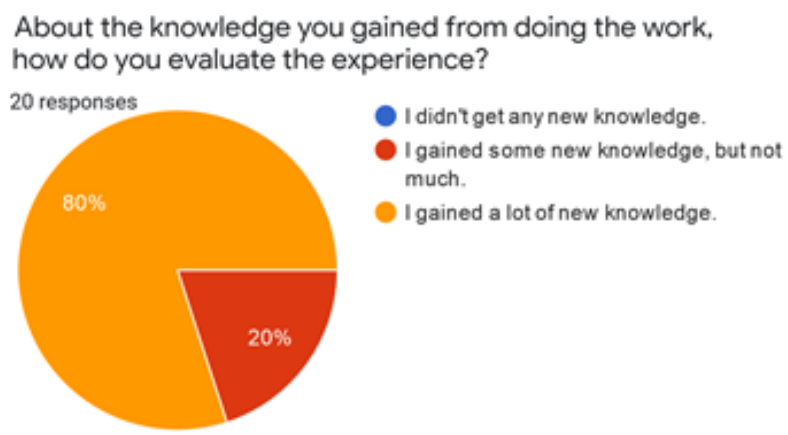

Figure 4: Knowledge obtained in carrying out the work.

The exciting thing about this result is that a very significant part of the students, about $80 \%$, stated that they obtained much new knowledge. The other party, $20 \%$, stated that they also gained new knowledge, although to a lesser extent. Nobody claimed to have learned anything new.

\subsection{About continuing to use the AWS Educate Program after the class}

Concerning this question, the goal was to determine if students intend to continue using the AWS Educate Program on other occasions, notably in other classes. Figure 5 shows the result.

In this sense, a little more than half of the respondents stated that they would use AWS 


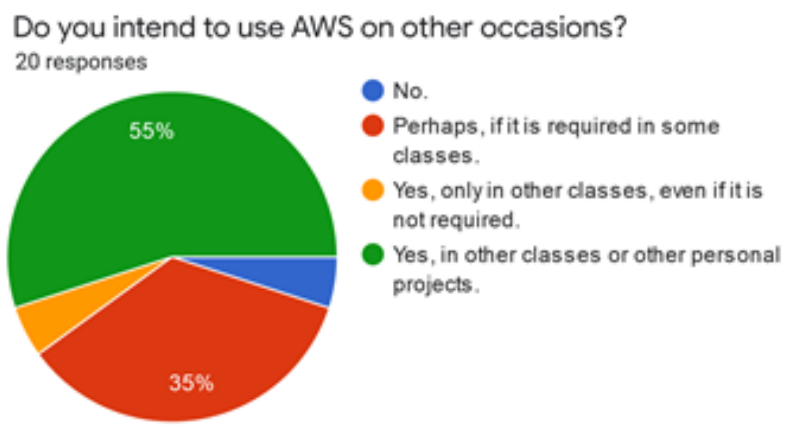

Figure 5: The usage of AWS Educate Program after the course.

in other circumstances, eventually even for personal projects. Another significant part, $35 \%$, could be used in another discipline if required. Only $5 \%$ (one student) said he would not want to use AWS.

\section{Discussion}

The results pointed to a good acceptance of the AWS Educate program and to the use of cloud computing resources to implement the work proposed in the discipline. In the qualitative section of the questionnaire, where respondents could express themselves freely, comments were positive.

The students considered that the incorporation of cloud computing concepts in the Operating Systems discipline brought a more updated view of the concepts covered in the discipline and prepared them for the technological challenges that they will experience in the market.

However, students faced some difficulties in implementing the work. Part of these difficulties is inherent to the problem that they had to solve in the proposed work. After all, it was necessary to seek strategies to solve the problem that was not very evident.

Most importantly, all students recognize that they have gained new knowledge to a greater or lesser extent. This new knowledge will undoubtedly be a differential in the training of these students.

Finally, at least half of the students said they plan to use AWS for other opportunities, including personal projects. One third intends to use, if required, in other disciplines only.

This result indicates that students recognize the potential of cloud computing, but this opinion is not unanimous. There is some resistance to using cloud computing resources. Probably because of the issues of remote access, security, and other concerns about using computational resources out of physical reach.

\section{Conclusion}

The experience of using computing resources in the AWS cloud by students in the Operating Systems class was successful. In general, students liked the experience and recognized that they acquired new knowledge despite some difficulties encountered.

However, the option to use the AWS computing cloud for other projects is not a consensus among students. The reason for this deserves further investigation. We suspect that the means of access and the necessary specifications to configure the environment in the cloud before its use is one of the reasons for the lack of motivation of some students using the computational cloud.

In any case, it is important to highlight that the AWS Educate program made a fundamental contribution so that students can be better trained for the job market. Besides, we hope that this report will motivate other professors to use the computing resources of the cloud in their subjects. The results presented here suggest that there are good chances of success in experiences with other classes.

\section{Acknowledgements}

The author thanks the AWS Educate Program for the credits for using the AWS cloud by students in the Operating Systems class. 